\title{
Infrared absorption spectra of hot ammonia
}

Christopher A. Beale*a, Robert J. Hargreaves ${ }^{b, c}$, Phillip Coles $^{d}$, Jonathan Tennyson ${ }^{d}$, and Peter F. Bernath ${ }^{b}$

* Corresponding author (cbeale@odu.edu)

a Department of Ocean, Earth and Atmospheric Sciences, Old Dominion University, Norfolk, VA, USA, 23527

${ }^{\mathrm{b}}$ Department of Chemistry and Biochemistry, Old Dominion University, Norfolk, VA, USA, 23527

c Atmospheric, Oceanic \& Planetary Physics, University of Oxford, Clarendon Laboratory, Parks Road, Oxford, OX1 3PU, UK

${ }^{d}$ Department of Physics and Astronomy, University College London, Gower Street, London WC1E 6BT, UK

(Note for production: this paper is for the HITRAN Special Issue)

\section{Abstract}

Infrared absorption spectra of $\mathrm{NH}_{3}$ have been obtained at high resolution $\left(0.02 \mathrm{~cm}^{-1}\right)$ at seven temperatures between 296 and $973 \mathrm{~K}$. The spectra were recorded using a Bruker IFS 125 infrared Fourier transform spectrometer in the $2400-5500 \mathrm{~cm}^{-1}$ region and empirical lower state energies have been obtained by comparison of line strengths at different temperatures. Using two reference line lists, quantum number assignments have been made for each temperature for between 1660 and 3020 transitions, with J up to 22 . The line lists obtained provide accurate line positions as well as intensities and experimental lower state energies at temperatures relevant for modeling the atmospheres of brown dwarfs and exoplanets.

Highlights

- Fourier transform infrared absorption spectra of hot ammonia

- Line positions, empirical lower state energies and intensities measured

- Line lists for brown dwarf and exoplanet atmospheres

\section{Keywords}

Fourier transform spectroscopy; infrared absorption; hot ammonia; brown dwarfs; line lists 


\section{Introduction}

Ammonia $\left(\mathrm{NH}_{3}\right)$ is a widely studied molecule in atmospheric chemistry. In the Earth's atmosphere, where sources include animal waste and fertilizers [1], it acts as a precursor in the production of aerosols [2] and particulate matter [3]. Deposition of atmospheric $\mathrm{NH}_{3}$ can lead to fertilization, a side effect of which may be decreased biodiversity [4].

$\mathrm{NH}_{3}$ has also been observed in a number of astrophysical environments. In the solar system, it has been detected in the atmospheres of Jupiter [5, 6], Saturn [7] and Titan [8] as well as on comets [9, 10]. The pure rotational transitions and inversion transitions of $\mathrm{NH}_{3}$ have been detected in molecular clouds [11, 12] making it one of the first extraterrestrial polyatomic molecules discovered. The atmospheres of cool astronomical objects, such as brown dwarfs and exoplanets, are of low enough temperatures to allow small molecules such as $\mathrm{NH}_{3}$ to form and maintain large enough concentrations to be detected.

Sub-stellar objects with sufficiently low mass ( $<0.08$ solar masses) are known as brown dwarfs and cannot fuse hydrogen within their cores [13]. The first such object discovered was Gliese 229B [14] and since then a large number of brown dwarfs have been detected with atmospheres cool enough ( $500-2400 \mathrm{~K})$ for a number of molecular species to exist. Indeed, brown dwarfs, similar to stars, are classified by the presence or absence of particular atomic and molecular features. The hottest such objects, the $L$ dwarfs, contain features from electronic transitions of metal hydrides such as $\mathrm{FeH}[15]$ and $\mathrm{CrH}[16,17]$. T dwarfs are distinguished by hot $\mathrm{H}_{2} \mathrm{O}$ and $\mathrm{CH}_{4}$ transitions $[18,19]$. $\mathrm{NH}_{3}$ is observed in late $\mathrm{T}$ dwarfs, with increasing concentrations as the objects cool $[19,20]$. As such, $\mathrm{NH}_{3}$ is expected to characterize a yet cooler class, the $\mathrm{Y}$ dwarfs that have an approximate maximum temperature of $\sim 700 \mathrm{~K}$. Y-dwarfs have now been observed and $\mathrm{NH}_{3}$ appears as a shoulder on a feature at $1.58 \mu \mathrm{m}[21,22]$.

Since the discovery of the first exoplanet in 1995 [23] more than 3500 have been discovered (http://exoplanet.eu/). If the planet passes in front of the star it orbits, then it may be detected by observation of periodic decreases in light intensity of the parent star. This technique is known as the transit method and was first used successfully to detect the planet HD 209458b [24] and has since been used by the Kepler mission to observe large numbers of exoplanet candidates [25]. This technique also allows the spectrum of the exoplanet atmosphere to be obtained by recording the transit dips as a function of wavelength [26] and use of the method has resulted in the detection of a number of molecules such as $\mathrm{H}_{2} \mathrm{O}[27,28], \mathrm{CH}_{4}$ [29], $\mathrm{CO}$ [30] and $\mathrm{CO}_{2}$ [31]. As yet, $\mathrm{NH}_{3}$ has not been observed in the atmosphere of an exoplanet, however a number of modelling studies have predicted its presence in hydrogen-rich hot-Jupiters [32-34].

$\mathrm{NH}_{3}$ is a polyatomic molecule having four atoms arranged in a trigonal pyramid structure with $\mathrm{C}_{3 \mathrm{v}}$ symmetry [35]. Ammonia has a complex infrared spectrum with 6 fundamental vibrational modes, two of which are doubly degenerate. The four fundamental vibrational frequencies are: the symmetric stretch at $3336.2 \mathrm{~cm}^{-1}\left(v_{1}, a_{1}\right)$, the symmetric bend at $932.5 \mathrm{~cm}^{-1}\left(v_{2}, a_{1}\right)$, the antisymmetric stretch at $3443.6 \mathrm{~cm}^{-1}$ $\left(v_{3}, e\right)$ and the antisymmetric bend at $1626.1 \mathrm{~cm}^{-1}\left(v_{4}, e\right)$.

There has been extensive work on the spectroscopy of ammonia in the infrared; for example experimental line lists have been obtained in the $2 \mu \mathrm{m}$ [36] and $3 \mu \mathrm{m}$ [37] regions. The most complete line assignments for $\mathrm{NH}_{3}$ in the infrared are compiled in the HITRAN 2012 database [38], however HITRAN is intended for 
applications near room temperature and lacks complete hot band coverage in this region [39]. The hot bands are essential when comparisons are made to high temperature atmospheres. A comprehensive theoretical line list, BYTe, has been calculated for $\mathrm{NH}_{3}$ that can be used at temperatures up to $1500 \mathrm{~K}$ and contains approximately 1.1 billion transitions [40]. The ro-vibrational calculations from Huang et al., [41, 42] and references therein, provide an additional line list for ammonia in this region. High temperature experimental line lists have been obtained using emission spectroscopy at high resolution $\left(0.01 \mathrm{~cm}^{-1}\right)$ from $740-2100 \mathrm{~cm}^{-1}$ [43] and $1650-4000 \mathrm{~cm}^{-1}$ [44]. Absorption spectra at moderate resolution $\left(0.09 \mathrm{~cm}^{-1}\right)$ of hot samples at atmospheric pressure have also been recorded in the $500-2100 \mathrm{~cm}^{-1}$ [45] and $2100-5500 \mathrm{~cm}^{-1}$ [46] spectral regions. Existing experimental line lists have been used in an energy level analysis (MARVEL) to predict a large number of unmeasured lines [47]. Our work reported below details an extension of these experimental line lists, in the $2400-5500 \mathrm{~cm}^{-1}$ region using an improved cell design and technique [48] to record transmission spectra of hot samples. We provide empirical lower state energies, intensities and line positions $\left( \pm 0.002 \mathrm{~cm}^{-1}\right)$ which can be used in atmospheric models for brown dwarfs and exoplanets.

\section{Experimental}

Transmission spectra were recorded at seven different temperatures, $23^{\circ} \mathrm{C}, 200^{\circ} \mathrm{C}, 300^{\circ} \mathrm{C}, 400^{\circ} \mathrm{C}, 500^{\circ} \mathrm{C}$, $600^{\circ} \mathrm{C}$ and $700{ }^{\circ} \mathrm{C}$, using a sealed quartz cell, tube furnace and Fourier transform spectrometer. This range includes the effective temperatures of the mid and late $\mathrm{T}$ dwarfs [19] and $\mathrm{Y}$ dwarfs [22] as well as the coolest observed hot Jupiters [32]. Above $700{ }^{\circ} \mathrm{C}$ thermal decomposition of the sample gas reduces the signal to such an extent that reliable spectra could not be obtained.

The transmission spectrum for each temperature is obtained from four individual spectra that, when combined, correct for $\mathrm{NH}_{3}$ and cell emission; this method has been used previously to record spectra for methane [48]. An absorption spectrum $\left(\mathrm{A}_{\mathrm{ab}}\right)$ is recorded with $\mathrm{NH}_{3}$ in the cell at temperature with an external emission source ( $200 \mathrm{~W}$ tungsten halogen broadband lamp). A background reference spectrum for the absorption $\left(A_{\text {ref }}\right)$ is recorded without the $\mathrm{NH}_{3}$ in the cell. The emission spectrum $\left(\mathrm{B}_{\mathrm{em}}\right)$ is recorded with $\mathrm{NH}_{3}$ in the cell at temperature without the lamp. The background reference spectrum for emission $\left(\mathrm{B}_{\mathrm{ref}}\right)$ is recorded without the sample in the cell and with the lamp turned off. These spectra are combined to calculate the transmission spectrum for each temperature as

$\tau=\frac{A_{a b}-B_{e m}}{A_{r e f}-B_{r e f}}$.

Spectra were recorded in the $2400-5500 \mathrm{~cm}^{-1}$ region, providing overlap with previous experimental work [44]. This previous work includes spectral regions that cover $3 v_{2} / v_{2}+v_{4}$ and $v_{1} / v_{3} / 2 v_{4}$ bands and the work provided here extends this coverage to include spectral regions that contain the $v_{1}+v_{2} / v_{2}+v_{3}$ and $v_{1}+v_{4} / v_{3}+v_{4}$ bands as well as associated hot bands.

The $50 \mathrm{~cm}$ quartz tube sample cell was used under static conditions to contain the $\mathrm{NH}_{3}$ sample. The cell is contained within the tube furnace which is heated to the appropriate temperature, which is accurate to within $\pm 10^{\circ} \mathrm{C}$. The cell was aligned with the entrance aperture of a Bruker IFS 125 HR Fourier transform spectrometer, and radiation was focused into the spectrometer using a $\mathrm{CaF}_{2}$ lens. The spectrometer used a $\mathrm{CaF}_{2}$ beamsplitter, covering the spectral region $1650-7000 \mathrm{~cm}^{-1}$, and an indium antimonide (InSb) detector. Experimental parameters are summarized in Table 1. 
The resulting transmittance spectra contained a number of $\mathrm{H}_{2} \mathrm{O}$ lines which were removed manually using the Bruker OPUS software. After combining the spectra at each temperature to calculate a transmission spectrum, peaks were picked using WSpectra [49] to measure their position and intensity. Lines were calibrated for line position and intensity by matching strong and isolated lines that are also found in the HITRAN 2012 database. The number of lines found for each temperature is given in Table 2.

The line intensity equation gives the line intensity as a function of temperature,

$$
S^{\prime}=\frac{2 \pi^{2} v_{10} S_{J^{\prime} J^{\prime \prime}}}{2 \varepsilon_{0} h c Q} \exp \left(-\frac{E^{\prime \prime}}{k T}\right)\left[1-\exp \left(\frac{h v_{10}}{k T}\right)\right]
$$

where $v_{10}$ is the line frequency, $S_{J^{\prime \prime} \prime}$ is the line strength, $\varepsilon_{0}$ is the permittivity of free space, $h$ is the Planck constant, $c$ is the speed of light, $Q$ is the internal partition function, $E^{\prime \prime}$ is the lower state energy, $k$ is the Boltzmann constant and $T$ is the temperature [35]. If a line is measured at different temperatures, the intensity of the line at each temperature may be compared to the intensity at a reference temperature by taking a ratio of the line intensity equation

$$
\frac{S^{\prime}}{S_{0}^{\prime}}=\frac{Q_{0}}{Q} \exp \left(\frac{E^{\prime \prime}}{k T_{0}}-\frac{E^{\prime \prime}}{k T}\right)\left[\frac{1-\exp \left(-\frac{h v_{10}}{k T}\right)}{1-\exp \left(-\frac{h v_{10}}{k T_{0}}\right)}\right]
$$

where $S_{0}^{\prime}$ and $T_{0}$ refer to the line intensity and temperature of the reference measurement. For these results $500{ }^{\circ} \mathrm{C}$ was used as the reference as this temperature contained the most lines with which to compare the line intensities of other temperatures. If there was no line measured at $500{ }^{\circ} \mathrm{C}$ then the reference used was the temperature with the next greatest number of lines. The partition function used for $\mathrm{NH}_{3}$ was obtained from Yurchenko et al. [40], which for our temperature range is essentially the same as the more recent values of Sousa-Silva et al. [41].

The lower state energy can be obtained from a rearranged version of the line intensity equation

$$
\ln \left(\frac{S Q}{S_{0} Q_{0}}\left[\frac{1-\exp \left(-\frac{h v_{10}}{k T}\right)}{1-\exp \left(-\frac{h v_{10}}{k T_{0}}\right)}\right]\right)=\frac{E^{\prime \prime}}{k T_{0}}-\frac{E^{\prime \prime}}{k T}
$$

where the intensity ratio on the left hand side can be plotted against $1 / k T$. Plotted for multiple temperatures, the gradient of this line gives the lower state energy.

\section{Results and Discussion}

Forty Torr of $\mathrm{NH}_{3}$ was used in the sample cell for these measurements in order to observe weaker transitions. However, at this pressure, many strong lines become saturated, particularly in the $Q$ branch of the $v_{1}$ fundamental mode and the lines are pressure-broadened. The resulting line lists are provided at each temperature (as supplementary material) and contain line position, line intensity and lower state energy (if calculated). For observed lines that could be assigned using the MARVEL or HITRAN line lists, the corresponding position, intensity, lower state energy and quantum number assignment is provided. Observed saturated lines, for which accurate positions and intensities could not be measured, were 
excluded and replaced with HITRAN lines with intensities greater than $2.0 \times 10^{-20} \mathrm{~cm}$ molecule $\mathrm{e}^{-1}$. The experimental positions were calibrated to strong lines in HITRAN, and the mean difference between all matched lines is approximately $4.0 \times 10^{-4} \mathrm{~cm}^{-1}$ with a standard deviation of $3.0 \times 10^{-4} \mathrm{~cm}^{-1}$ after calibration. Similarly, the accuracy of the intensities of the experimentally obtained lines is measured by comparison with matched HITRAN lines; for these the mean difference is within $20 \%$.

The top panels of Figures 1 and 2 show overviews of the recorded spectra at 296 and $773 \mathrm{~K}$, respectively. The middle panels show simulated spectra from the BYTe line list and the bottom panels similarly show simulated spectra from the HITRAN line list. The spectral simulation uses the Reference Forward Model [50], a line-by-line radiative transfer model, to calculate the transmission through a $50 \mathrm{~cm}$ cell with the experimental pressure and temperature. Individual line broadening parameters for the lines in BYTe were obtained as averages from HITRAN 2012 and applied to all lines. The HITRAN spectral simulation uses the line positions and intensities from HITRAN 2012 as well as the averaged broadening parameters applied globally, therefore allowing for comparisons between HITRAN, BYTe and our measurements.

The experimental spectrum at $296 \mathrm{~K}$ (Figure 1) shows some saturated lines of the $v_{1}$ fundamental, $v_{1}+v_{2} / v_{2}+v_{3}$ and $v_{1}+v_{4} / v_{3}+v_{4}$ modes which have been replaced in the final line list. In general, there is good agreement between the three spectra at $296 \mathrm{~K}$. For $773 \mathrm{~K}$, the experimental and BYTe spectra in Figure 2 clearly show a number of additional hot bands that are not present in the HITRAN spectrum, most notably the Q-branch of the $v_{1}+3 v_{3}-3 v_{3}$ band at around $3850 \mathrm{~cm}^{-1}$ and the $v_{2}+v_{3}-v_{2}$ band from $4000 \mathrm{~cm}^{-1}$ to 4200 $\mathrm{cm}^{-1}$. Other hot bands are included in the $3000-3600 \mathrm{~cm}^{-1}$ regions and $4200-4700 \mathrm{~cm}^{-1}$ regions although they are not shown because of the density of lines. Some lines in HITRAN have an incorrect lower state energy which can be seen clearly in Figure 2 at, for example, around $4150 \mathrm{~cm}^{-1}$ where an error in lower state energy results in a 'bad' extrapolation with temperature.

A more detailed view of short segments of the three spectra is shown in Figures 3 and 4. Figure 3 again shows the three spectra for $296 \mathrm{~K}$. There are a number of lines that assignable in both the experimental and BYTe spectra; one example being the strong line at $4151.8 \mathrm{~cm}^{-1}$ (the $R(6)$ line of the $v_{1}+v_{2}$ band) that appears in all three spectra within $0.1 \mathrm{~cm}^{-1}$ with a similar intensity. Even at room temperature, however, there are a number of lines in the experimental spectrum that are missing from HITRAN and a number of inconsistencies between the experimental spectrum and the BYTe simulation. Lines in the experimental line lists which have been matched to lines in either reference line list are marked by an asterisk. At 773 $\mathrm{K}$, the hot lines in the experimental spectrum at $4141.0 \mathrm{~cm}^{-1}, 4147.4 \mathrm{~cm}^{-1}$ and $4153.2 \mathrm{~cm}^{-1}$ can be matched to lines in the BYTe spectrum, although their positions are shifted. In this region the shift is over $1 \mathrm{~cm}^{-1}$ towards higher wavenumbers, although in other regions the shift value and direction vary. While the strong lines can be identified, it becomes more problematic to assign weaker features from BYTe primarily due to the observed shifts. The lines in Figure 4 which are not matched (i.e., those without an asterisk) include a number of strong lines, and these are mostly hot lines. This demonstrates the difficulty in assigning lines in regions with overlapping hot bands where the line position from calculated spectra is of insufficient accuracy.

The empirical lower state energies are plotted against line position in Figure 5 in the lower panel along with those from HITRAN in the upper panel. The near-vertical lines are belong to $Q$ branches and the parabolic features the left and right of the $Q$-branches are from the $P$ and $R$ branches, respectively.

There are a number of notable differences between the HITRAN lower state energies and those obtained by experiment (Fig 5). The incompleteness of HITRAN can be seen between 3600 and $4100 \mathrm{~cm}^{-1}$, 
experimental data has been obtained in this region. There are also very few lower state energy values listed in HITRAN with energies above $2000 \mathrm{~cm}^{-1}$; some of these are from fundamental and combination transitions and can be seen in the bottom panel, particularly the $Q$ branch of the $V_{1}$ band and the $P$ and $R$ branches of the $V_{1}+v_{2}$ band. However, most of the lines with high lower state energies are from hot transitions. The most notable 'hot' band in the lower panel is the strong $Q$ branch at a position $3850 \mathrm{~cm}^{-}$ 1 , extending from a lower state energy of $2000-3000 \mathrm{~cm}^{-1}$, which is not provided in HITRAN. This feature can be seen in the $773 \mathrm{~K} \mathrm{NH}_{3}$ spectra in Figure 2 in both the experimental data (top) and BYTe (center) panels and not in the HITRAN (lower) panel.

The MARVEL analysis of $\mathrm{NH}_{3}$ [47] and re-analysis of the HITRAN $\mathrm{NH}_{3}$ database [39] are line lists in the region of interest which contain quantum number assignments. MARVEL provides a self-consistent list of fully assigned line positions, but no associated line intensities. Therefore, rather than using MARVEL directly, we used the MARVEL adjusted BYTe line list BARVEL, which was computed by replacing BYTe energies with the corresponding values from MARVEL. Disregarding the $13 \%$ of MARVEL states that could not be matched to corresponding BYTe states, BARVEL provides intensity data for roughly 18,500 (66\%) of MARVEL transitions, plus many predicted transitions between MARVEL energy levels that have not been observed experimentally. The remaining line list consists of transitions for which either one or both of the states takes its energy from BYTe. We decided to only use the transitions for which both upper and lower state had their values taken from MARVEL to avoid any discrepancies in line positions due to the inaccuracy of BYTe.

The positions and intensities from these lists may be compared to those obtained experimentally to provide tentative assignments. Lines with positions within $0.002 \mathrm{~cm}^{-1}$ and intensities between 0.5 and 2.0 times that of the experimental lines were considered matched, with the number of assigned lines from each reference list given in Table 2, it should be noted that many of these assignments are for the same lines at different temperatures. Figure 6 shows the comparison of lower state energies from matched lines.

\section{Conclusions}

High resolution transmission spectra of ammonia recorded at seven temperatures between $22{ }^{\circ} \mathrm{C}$ and 700 ${ }^{\circ} \mathrm{C}$ were used to calculate lower state energies in the $2400-5500 \mathrm{~cm}^{-1}$ region. These spectra include several hot bands which are not present in HITRAN even at room temperature with tentative assignments of J up to 22. Therefore, this work currently provides the most accurate line list for simulating room temperature (and higher) $\mathrm{NH}_{3}$ observations in this spectral region. Extrapolating room temperature HITRAN results to higher temperatures results in a substantial decrease in total intensity as the hot bands are not included (Table 4) and this work provides intensity estimates for the hot bands in this region.

Calculated line lists such as BYTe [40] provide more lines than are experimentally observable due weakness of millions of lines in the spectrum and the sensitivity required to measure them. Calculations give a much more complete total intensity sum over multiple bands, however, positions of individual lines in BYTe are less well determined than in the experiment measurements.

The experimental line lists obtained can be used directly in atmospheric models for brown dwarfs and exoplanets. The line lists can also be used to create template spectra for cross correlation with high 
resolution spectra of planetary systems. The cross correlation method for detecting molecules on exoplanets, such as those used to detect CO on HD 209458 [51] by analyzing Doppler-shifted spectral features of a transiting exoplanet, require high resolution template spectra with accurate line positions and intensities. The line lists of this work satisfy both the high temperature and high resolution requirements for current exoplanetary research.

Table 1: Experimental conditions

\begin{tabular}{ll}
\hline Parameter & Value \\
\hline Spectral region $\left(\mathrm{cm}^{-1}\right)$ & $2400-5500$ \\
Detector & $\mathrm{InSb}$ \\
Beamsplitter & $\mathrm{CaF}_{2}$ \\
Spectrometer Windows & $\mathrm{CaF}_{2}$ \\
Lens & $\mathrm{CaF}_{2}$ \\
Scans & 300 \\
Resolution $\left(\mathrm{cm}^{-1}\right)$ & 0.01 \\
$\mathrm{NH}_{3}$ Pressure (Torr) & 40 \\
Zerofilling factor & $\times 16$ \\
\hline
\end{tabular}

Table 2: Total number lines, the number of added lines, partition functions and tentative assignments made via comparison with each line list at each temperature [40]

\begin{tabular}{llllll}
\hline $\begin{array}{l}\text { Temperature } \\
(\text { K })\end{array}$ & $\begin{array}{l}\text { Number } \\
\text { of lines }\end{array}$ & $\begin{array}{l}\text { HITRAN } \\
\text { additions }\end{array}$ & $\begin{array}{l}\text { Partition } \\
\text { function }\end{array}$ & $\begin{array}{l}\text { Down et al. } \\
\text { assignments }\end{array}$ & $\begin{array}{l}\text { MARVEL } \\
\text { assignments }\end{array}$ \\
\hline 296 & 8494 & 722 & 1769.563 & 2136 & 1813 \\
473 & 16019 & 789 & 3792.440 & 3020 & 2721 \\
573 & 20300 & 825 & 5320.817 & 2976 & 2839 \\
673 & 23010 & 834 & 7252.482 & 2913 & 2906 \\
773 & 20066 & 713 & 9705.249 & 2569 & 2594 \\
873 & 18794 & 691 & 12818.840 & 2296 & 2346 \\
973 & 9985 & 598 & 16759.410 & 1660 & 1178 \\
\hline
\end{tabular}




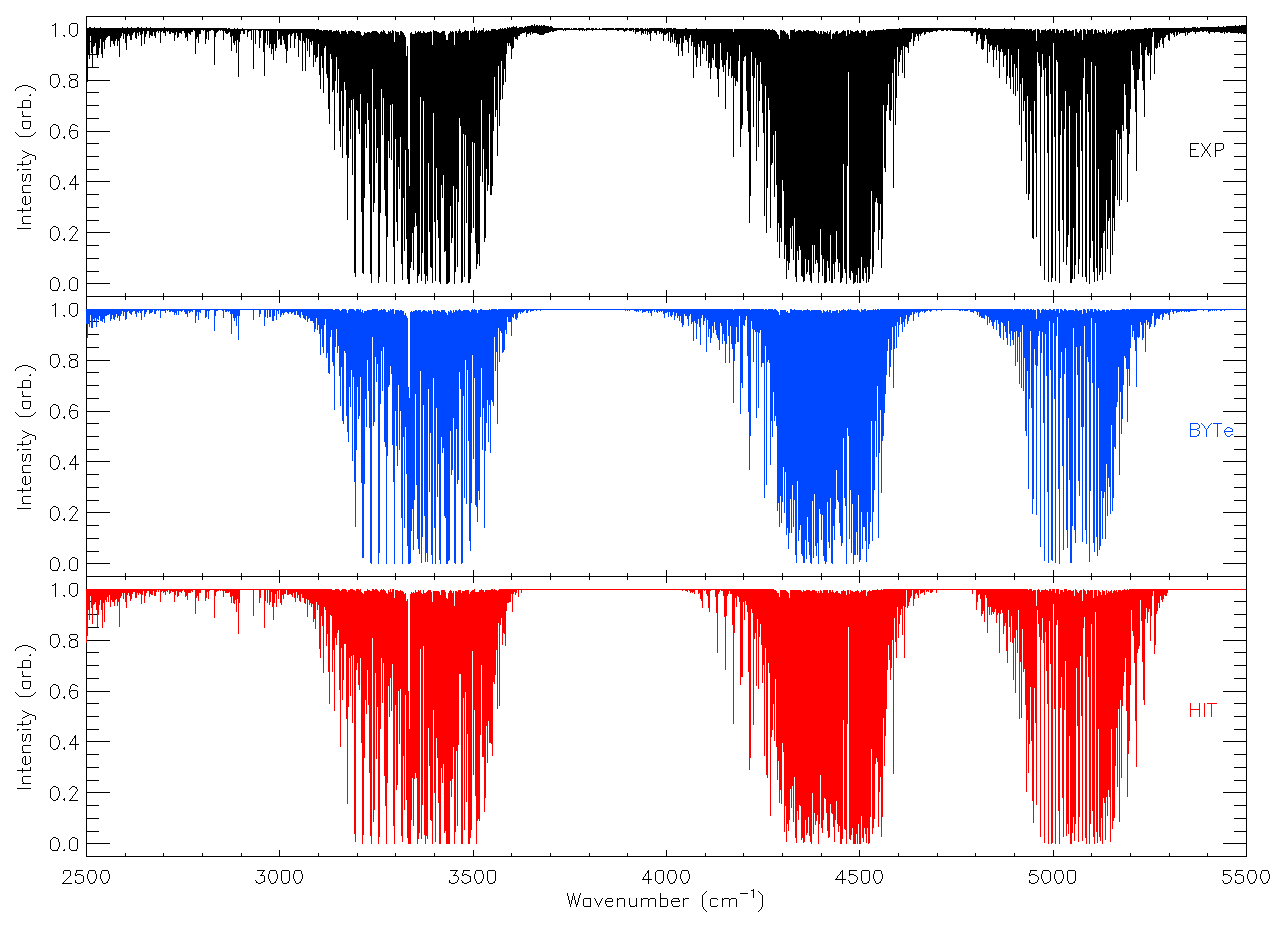

Figure 1: Overview of spectra of $\mathrm{NH}_{3}$ showing experimental data (top panel) and simulated spectra created to match the conditions of the experiment from the BYTe (center panel) and HITRAN (lower panel) line lists at $296 \mathrm{~K}$.

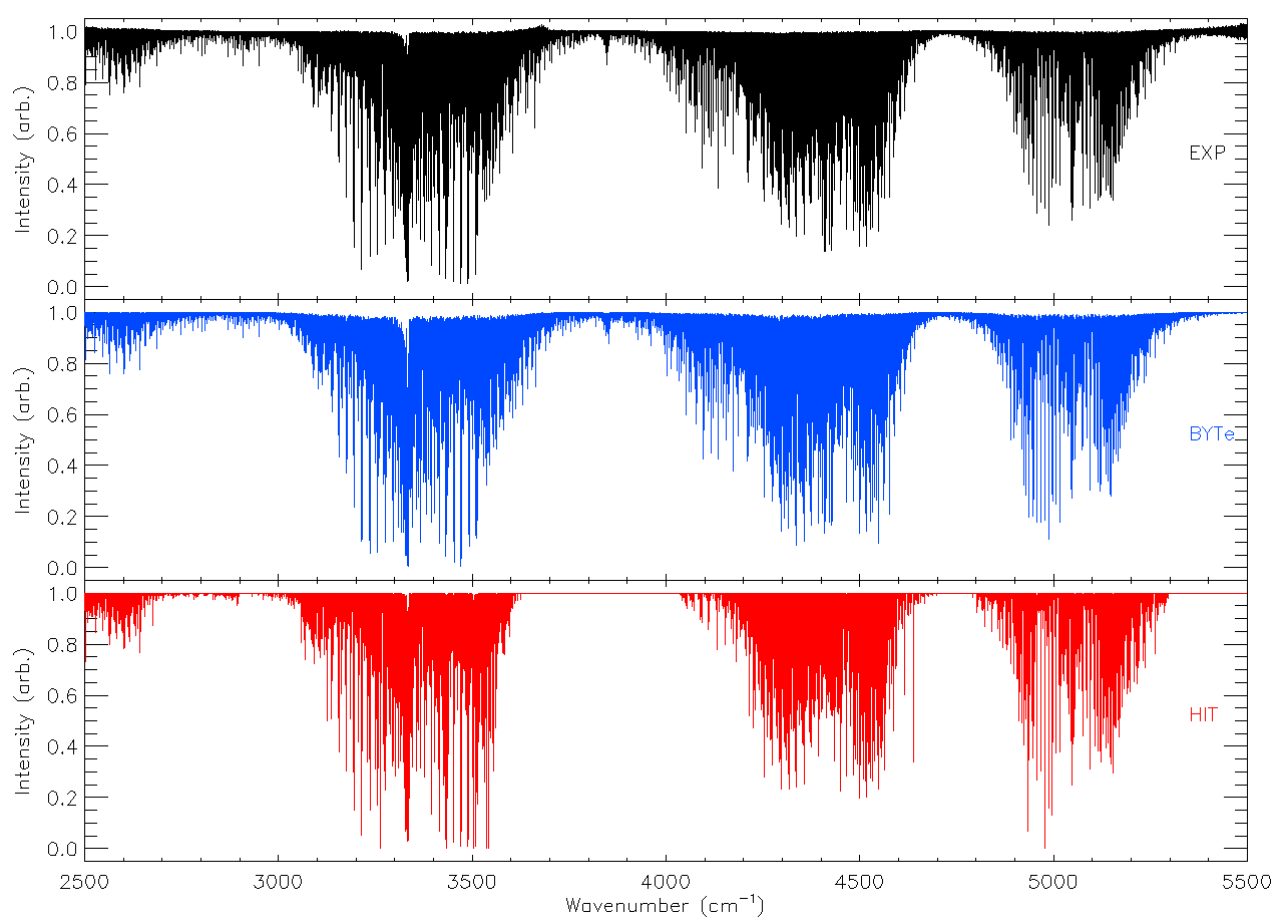


Figure 2: Overview of spectra of $\mathrm{NH}_{3}$ showing experimental data (top panel) and simulated spectra created to match the conditions of the experiment from the BYTe (center panel) and HITRAN (lower panel) line lists at $773 \mathrm{~K}$.

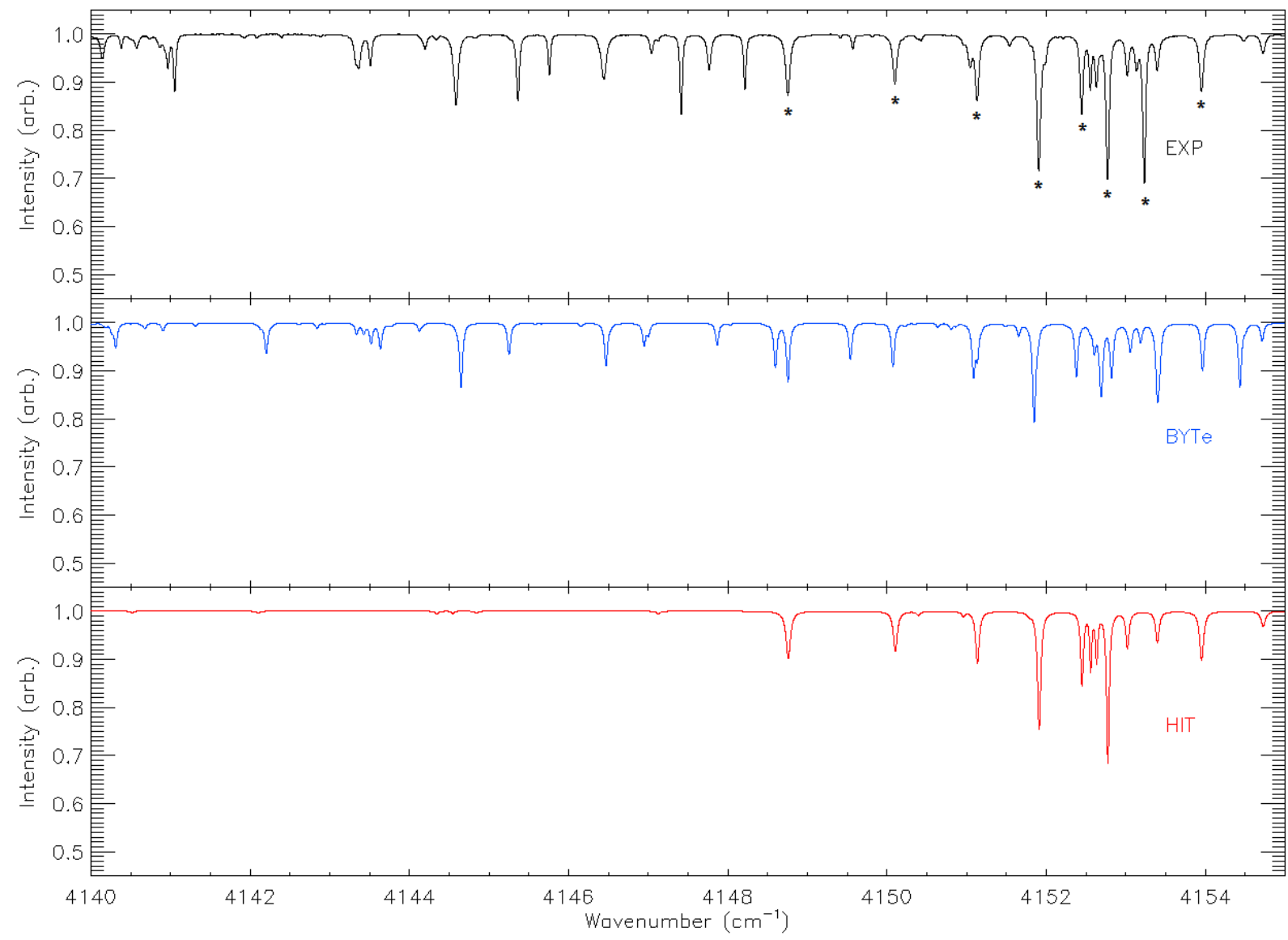

Figure 3: Detailed spectra of $\mathrm{NH}_{3}$ showing experimental data (top panel) and simulated spectra created to match the conditions of the experiment from the BYTe (center panel) and HITRAN (lower panel) line lists at $296 \mathrm{~K}$. Experimental lines that have been assigned by matching are marked by asterisks. 


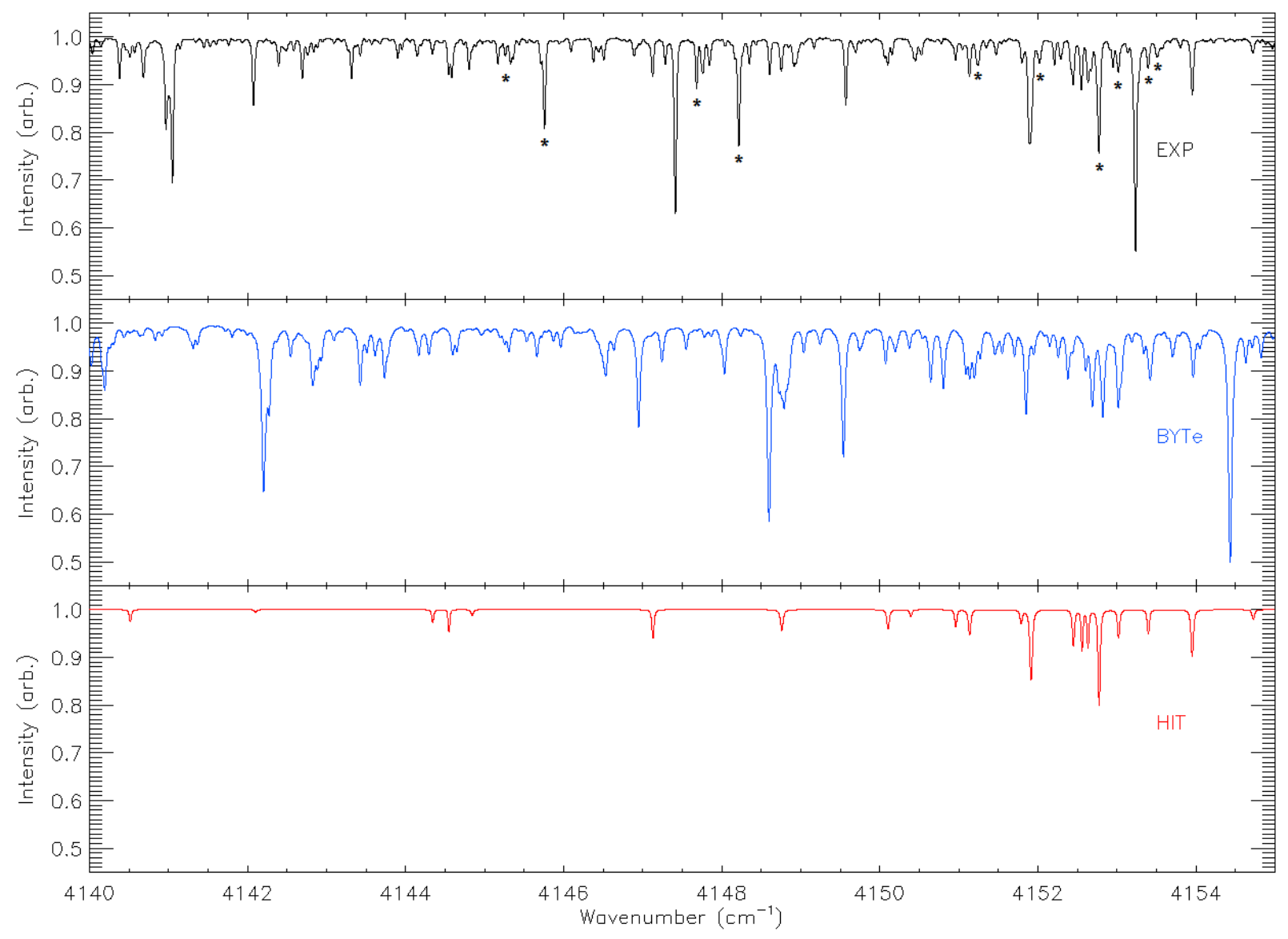

Figure 4: Detailed spectra of $\mathrm{NH}_{3}$ showing experimental data (top panel) and simulated spectra created to match the conditions of the experiment from the BYTe (center panel) and HITRAN (lower panel) line lists at $773 \mathrm{~K}$. Experimental lines that have been assigned by matching are marked by asterisks. 

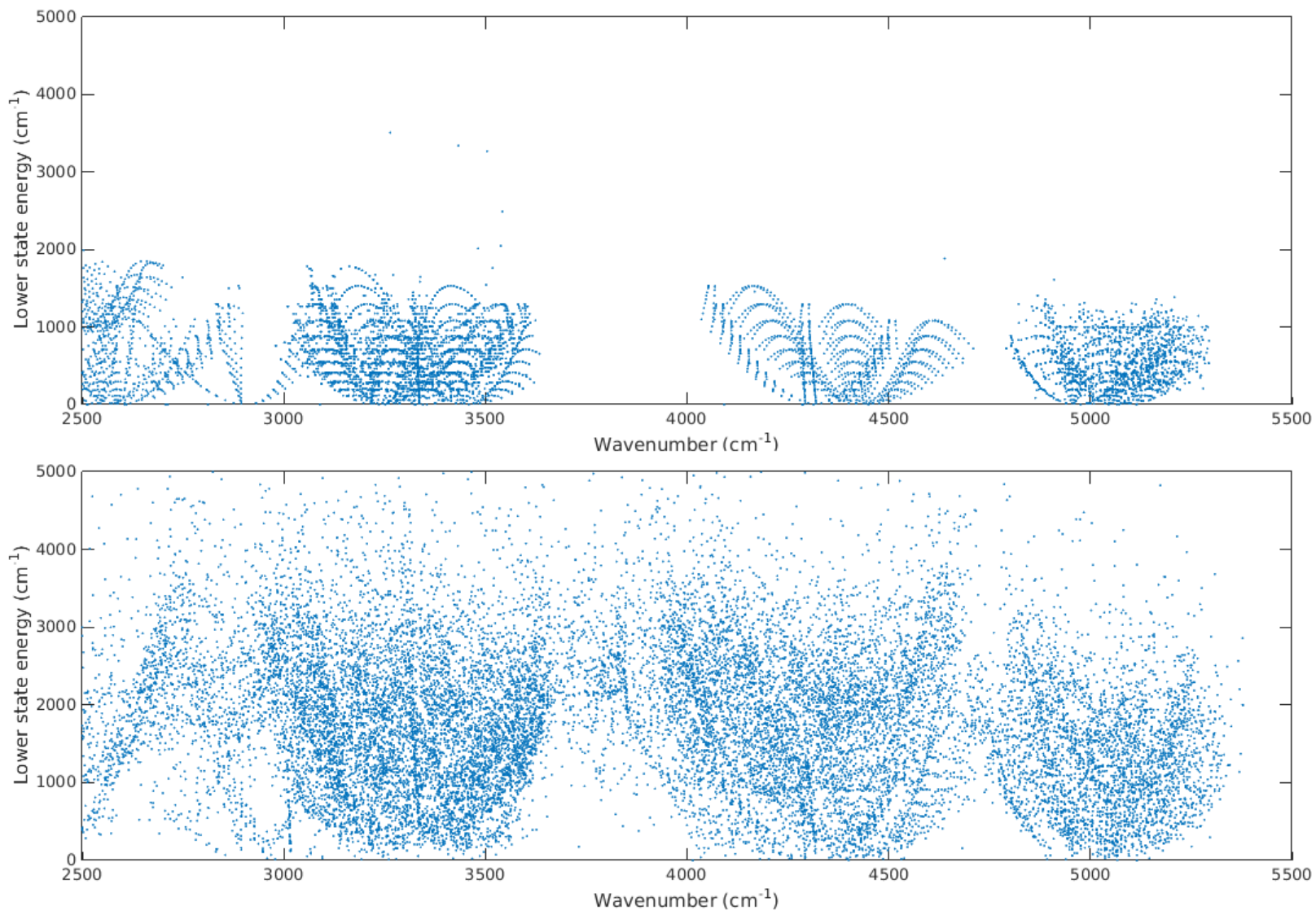

Figure 5: Comparison of lower state energies from the HITRAN line list [38] (top panel) and from the experimental line list (lower panel) as a function of line position. 


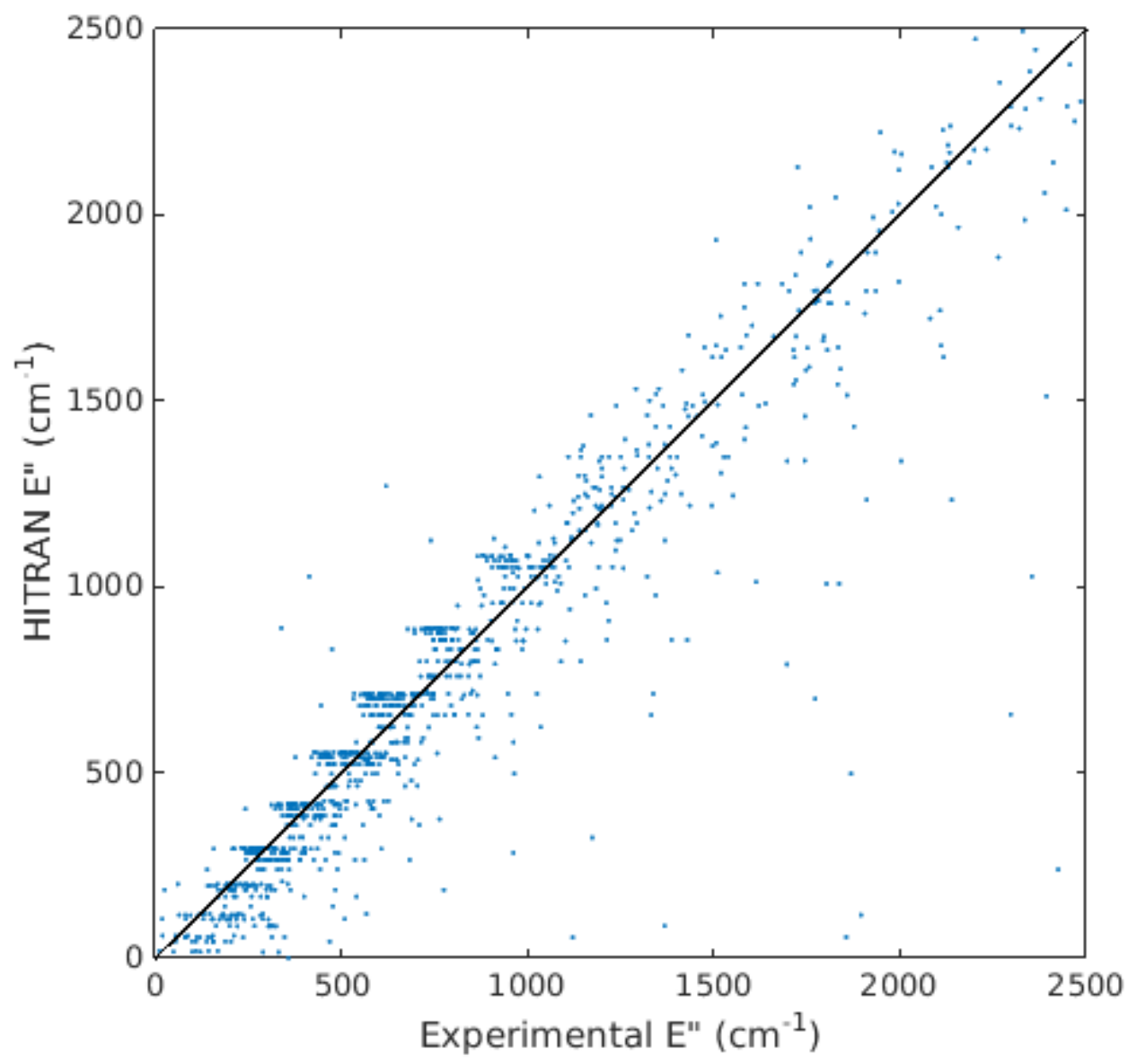

Figure 5: Comparison of matched lower state energies obtained from experiment ( $x$-axis) and the HITRAN re-analysis of Down et al. [39] (y-axis).

\section{Acknowledgements}

Support for this work was provided by the NASA laboratory astrophysics program.

\section{References}

[1] Beer R, Shephard MW, Kulawik SS, Clough SA, Eldering A, Bowman KW, et al. First satellite observations of lower tropospheric ammonia and methanol. Geophysical Research Letters. 2008;35:L09801

[2] Höpfner M, Volkamer R, Grabowski U, Grutter M, Orphal J, Stiller G, et al. First detection of ammonia (NH3) in the Asian summer monsoon upper troposphere. Atmos Chem Phys. 2016;16:14357-69.

[3] Clarisse L, Clerbaux C, Dentener F, Hurtmans D, Coheur P-F. Global ammonia distribution derived from infrared satellite observations. Nature Geosci. 2009;2:479-83.

[4] von Bobrutzki K, Braban CF, Famulari D, Jones SK, Blackall T, Smith TEL, et al. Field inter-comparison of eleven atmospheric ammonia measurement techniques. Atmos Meas Tech. 2010;3:91-112. 
[5] Wildt R. Ultrarote Absorptionsbande in den Spektren der Grossen Planeten. Naturwissenschaften. 1931;19.

[6] Carlson R, Smythe W, Baines K, Barbinis E, Becker K, Burns R, et al. Near-Infrared Spectroscopy and Spectral Mapping of Jupiter and the Galilean Satellites: Results from Galileo's Initial Orbit. Science. 1996;274:385-8.

[7] Fletcher LN, Swinyard B, Salji C, Polehampton E, Fulton T, Sidher S, et al. Sub-millimetre spectroscopy of Saturn's trace gases from Herschel/SPIRE. A\&A. 2012;539:A44.

[8] Nelson RM, Kamp LW, Matson DL, Irwin PGJ, Baines KH, Boryta MD, et al. Saturn's Titan: Surface change, ammonia, and implications for atmospheric and tectonic activity. Icarus. 2009;199:429-41.

[9] Bird MK, Janardhan P, Wilson TL, Huchtmeier WK, Gensheimer P, Lemme C. K-band Radio Observations of Comet Hale-Bopp: Detections of Ammonia and (Possibly) Water. Earth, Moon, and Planets. 1997;78:21-8.

[10] Biver N, Crovisier J, Bockelée-Morvan D, Szutowicz S, Lis DC, Hartogh P, et al. Ammonia and other parent molecules in comet 10P/Tempel 2 from Herschel/HIFl and ground-based radio observations. A\&A. 2012;539:A68.

[11] Cheung AC, Rank DM, Townes CH, Thornton DD, Welch WJ. Detection of N\$\{\mathrm $\{H\}\} \_\{3\} \$$ Molecules in the Interstellar Medium by Their Microwave Emission. Physical Review Letters.

1968;21:1701-5.

[12] Ho PTP, Townes CH. Interstellar Ammonia. Annual Review of Astronomy and Astrophysics. 1983;21:239-70.

[13] Burrows A, Hubbard WB, Lunine JI, Liebert J. The theory of brown dwarfs and extrasolar giant planets. Reviews of Modern Physics. 2001;73:719-65.

[14] Oppenheimer BR, Kulkarni SR, Matthews K, Nakajima T. Infrared Spectrum of the Cool Brown Dwarf GI 229B. Science. 1995;270:1478-9.

[15] Hargreaves RJ, Hinkle KH, Jr CWB, Wende S, Seifahrt A, Bernath PF. High-resolution $1.6 \mu$ m Spectra of FeH in M and L Dwarfs. The Astronomical Journal. 2010;140:919.

[16] Burrows A, Ram RS, Bernath P, Sharp CM, Milsom JA. New CrH Opacities for the Study of L and Brown Dwarf Atmospheres. The Astrophysical Journal. 2002;577:986.

[17] Kirkpatrick JD, Reid IN, Liebert J, Cutri RM, Nelson B, Beichman CA, et al. Dwarfs Cooler than "M": The Definition of Spectral Type "L" Using Discoveries from the 2-Micron All-Sky Survey (2MASS). The Astrophysical Journal. 1999;519:802.

[18] Burgasser AJ, Geballe TR, Leggett SK, Kirkpatrick JD, Golimowski DA. A Unified Near-Infrared Spectral Classification Scheme for T Dwarfs. The Astrophysical Journal. 2006;637:1067.

[19] Cushing MC, Roellig TL, Marley MS, Saumon D, Leggett SK, Kirkpatrick JD, et al. A Spitzer Infrared Spectrograph Spectral Sequence of M, L, and T Dwarfs. The Astrophysical Journal. 2006;648:614. 
[20] Line MR, Teske J, Burningham B, Fortney JJ, Marley MS. Uniform Atmospheric Retrieval Analysis of Ultracool Dwarfs. I. Characterizing Benchmarks, GI 570D and HD 3651B. The Astrophysical Journal. 2015;807:183.

[21] Leggett SK, Tremblin P, Saumon D, Marley MS, Caroline VM, Amundsen DS, et al. Near-infrared Spectroscopy of the YO WISEP J173835.52+273258.9 and the Y1 WISE J035000.32-565830.2: The Importance of Non-equilibrium Chemistry. The Astrophysical Journal. 2016;824:2.

[22] Cushing MC, Kirkpatrick JD, Gelino CR, Griffith RL, Skrutskie MF, Mainzer A, et al. The Discovery of Y Dwarfs using Data from the Wide-field Infrared Survey Explorer (WISE). The Astrophysical Journal. 2011;743:50.

[23] Mayor M, Queloz D. A Jupiter-mass companion to a solar-type star. Nature. 1995;378:355-9.

[24] Charbonneau D, Brown TM, Latham DW, Mayor M. Detection of Planetary Transits Across a Sun-like Star. The Astrophysical Journal Letters. 2000;529:L45.

[25] Batalha NM, Rowe JF, Bryson ST, Barclay T, Burke CJ, Caldwell DA, et al. Planetary Candidates Observed by Kepler. III. Analysis of the First 16 Months of Data. The Astrophysical Journal Supplement Series. 2013;204:24.

[26] Bernath PF. Molecular opacities for exoplanets. Philosophical Transactions of the Royal Society A: Mathematical, Physical and Engineering Sciences. 2014;372.

[27] Barman TS. On the Presence of Water and Global Circulation in the Transiting Planet HD 189733b. The Astrophysical Journal Letters. 2008;676:L61.

[28] Tinetti G, Vidal-Madjar A, Liang M-C, Beaulieu J-P, Yung Y, Carey S, et al. Water vapour in the atmosphere of a transiting extrasolar planet. Nature, 2007;448:169-171.

[29] Swain MR, Vasisht G, Tinetti G. The presence of methane in the atmosphere of an extrasolar planet. Nature. 2008;452:329-31.

[30] Swain MR, Vasisht G, Tinetti G, Bouwman J, Chen P, Yung Y, et al. Molecular Signatures in the NearInfrared Dayside Spectrum of HD 189733b. The Astrophysical Journal Letters. 2009;690:L114.

[31] Swain MR, Tinetti G, Vasisht G, Deroo P, Griffith C, Bouwman J, et al. Water, Methane, and Carbon Dioxide Present in the Dayside Spectrum of the Exoplanet HD 209458b. The Astrophysical Journal. 2009;704:1616.

[32] Line MR, Vasisht G, Chen P, Angerhausen D, Yung YL. Thermochemical and Photochemical Kinetics in Cooler Hydrogen-dominated Extrasolar Planets: A Methane-poor GJ436b? The Astrophysical Journal. 2011;738:32.

[33] Burrows AS. Spectra as windows into exoplanet atmospheres. Proceedings of the National Academy of Sciences. 2014;111:12601-9.

[34] Venot O, Hébrard E, Agúndez M, Dobrijevic M, Selsis F, Hersant F, et al. A chemical model for the atmosphere of hot Jupiters. A\&A. 2012;546:A43.

[35] Bernath P. Spectra of Atoms and Molecules. 3 ed. New York: Oxford University Press; 2016. 
[36] Brown LR, Margolis JS. Empirical line parameters of NH3 from 4791 to $5294 \mathrm{~cm}-1$. Journal of Quantitative Spectroscopy and Radiative Transfer. 1996;56:283-94.

[37] Kleiner I, Brown LR, Tarrago G, Kou QL, Picqué N, Guelachvili G, et al. Positions and Intensities in the $2 v_{4} / v_{1} / v_{3}$ Vibrational System of ${ }^{14} \mathrm{NH}_{3}$ Near $3 \mu \mathrm{m}$. Journal of Molecular Spectroscopy. 1999;193:46-71.

[38] Rothman LS, Gordon IE, Babikov Y, Barbe A, Chris Benner D, Bernath PF, et al. The HITRAN2012 molecular spectroscopic database. Journal of Quantitative Spectroscopy and Radiative Transfer. 2013;130:4-50.

[39] Down MJ, Hill C, Yurchenko SN, Tennyson J, Brown LR, Kleiner I. Re-analysis of ammonia spectra: Updating the HITRAN ${ }^{14} \mathrm{NH}_{3}$ database. Journal of Quantitative Spectroscopy and Radiative Transfer. 2013;130:260-72.

[40] Yurchenko SN, Barber RJ, Tennyson J. A variationally computed line list for hot NH3. Monthly Notices of the Royal Astronomical Society. 2011;413:1828-34.

[41] Sousa-Silva C, Hesketh N, Yurchenko SN, Hill C, Tennyson J, High Temperature partition functions and thermodynamic data for ammonia and phosphine. Journal of Quantitative Spectroscopy and Radiative Transfer. 2014;142:66-74

[42] Huang X, Schwenke DW, Lee TJ. Rovibrational spectra of ammonia. I. Unprecedented accuracy of a potential energy surface used with nonadiabatic corrections. The Journal of Chemical Physics.

2011;134:044320.

[43] Huang X, Schwenke DW, Lee TJ. Rovibrational spectra of ammonia. II. Detailed analysis, comparison, and prediction of spectroscopic assignments for ${ }^{14} \mathrm{NH}_{3},{ }^{15} \mathrm{NH}_{3}$, and ${ }^{14} \mathrm{ND}_{3}$. The Journal of Chemical Physics. 2011;134:044321.

[44] Hargreaves RJ, Li G, Bernath PF. Hot $\mathrm{NH}_{3}$ Spectra for Astrophysical Applications. The Astrophysical Journal. 2011;735:111.

[45] Hargreaves RJ, Li G, Bernath PF. Ammonia line lists from 1650 to $4000 \mathrm{~cm}^{-1}$. Journal of Quantitative Spectroscopy and Radiative Transfer. 2012;113:670-9.

[46] Barton EJ, Yurchenko SN, Tennyson J, Clausen S, Fateev A. High-resolution absorption measurements of $\mathrm{NH}_{3}$ at high temperatures: $500-2100 \mathrm{~cm}^{-1}$. Journal of Quantitative Spectroscopy and Radiative Transfer. 2015;167:126-34.

[47] Barton EJ, Yurchenko SN, Tennyson J, Clausen S, Fateev A. High-resolution absorption measurements of $\mathrm{NH}_{3}$ at high temperatures: $2100-5500 \mathrm{~cm}^{-1}$. Journal of Quantitative Spectroscopy and Radiative Transfer. 2017;189:60-65.

[48] Al Derzi AR, Furtenbacher T, Tennyson J, Yurchenko SN, Császár AG. MARVEL analysis of the measured high-resolution spectra of ${ }^{14} \mathrm{NH}_{3}$. Journal of Quantitative Spectroscopy and Radiative Transfer. 2015;161:117-30.

[49] Hargreaves RJ, Bernath PF, Bailey J, Dulick M. Empirical Line Lists and Absorption Cross Sections for Methane at High Temperatures. The Astrophysical Journal. 2015;813:12. 
[50] Carleer MR. WSpectra: a Windows program to accurately measure the line intensities of highresolution Fourier transform spectra. 2001. p. 337-42.

[51] Dudhia A. The Reference Forward Model (RFM). Journal of Quantitative Spectroscopy and Radiative Transfer. Journal of Quantitative Spectroscopy and Radiative Transfer. 2017;186:243-253

[52] Snellen IAG, de Kok RJ, de Mooij EJW, Albrecht S. The orbital motion, absolute mass and highaltitude winds of exoplanet HD 209458b. Nature. 2010;465:1049-51. 